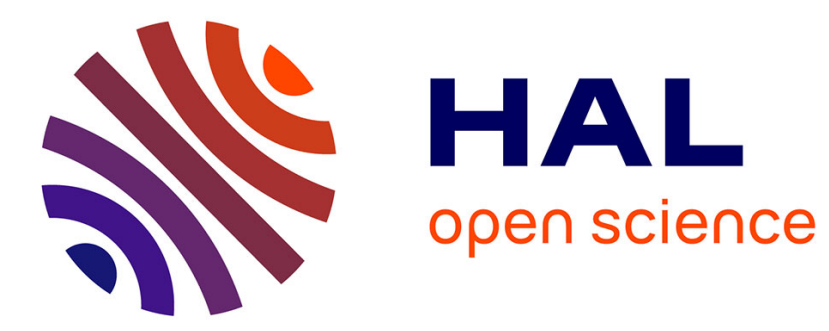

\title{
Intermediate filaments
}

Gaëlle Dutour-Provenzano, Sandrine Etienne-Manneville

\section{To cite this version:}

Gaëlle Dutour-Provenzano, Sandrine Etienne-Manneville. Intermediate filaments. Current Biology CB, 2021, 31 (10), pp.R522-R529. 10.1016/j.cub.2021.04.011 . pasteur-03273350

\section{HAL Id: pasteur-03273350 \\ https://hal-pasteur.archives-ouvertes.fr/pasteur-03273350}

Submitted on 24 Nov 2021

HAL is a multi-disciplinary open access archive for the deposit and dissemination of scientific research documents, whether they are published or not. The documents may come from teaching and research institutions in France or abroad, or from public or private research centers.
L'archive ouverte pluridisciplinaire HAL, est destinée au dépôt et à la diffusion de documents scientifiques de niveau recherche, publiés ou non, émanant des établissements d'enseignement et de recherche français ou étrangers, des laboratoires publics ou privés.

\section{다)(1)}

Distributed under a Creative Commons Attribution - NonCommercial| 4.0 International 


\section{Intermediate filaments in short}

Gaëlle Dutour-Provenzano ${ }^{1,2}$ and Sandrine Etienne-Manneville ${ }^{1}$

${ }^{1}$ Cell Polarity, Migration and Cancer Unit, Institut Pasteur, UMR3691 CNRS, Équipe Labellisée Ligue Contre le Cancer, F-75015, Paris, France.

${ }^{2}$ Sorbonne Université, Collège doctoral, F-75005 Paris, France.

Correspondence: setienne@pasteur.fr

\section{Summary}

Cell morphology, architecture and dynamics primarily relies on intracellular cytoskeletal networks, which in metazoans is mainly composed of actin microfilaments, microtubules and intermediate filaments (IFs). The diameter size of $10 \mathrm{~nm}$, intermediate between that of actin and of microtubules initially gave their names to IFs. However, IF structure, dynamics, mechanical properties and functions are not intermediate but set them apart from microfilaments and microtubules. Because of their nucleotide-independent assembly, the lack of intrinsic polarity, their relative stability and their complex composition, IFs have long been overlooked by cell biologists. The numerous human diseases associated with IF gene mutations, and the accumulating evidence of IF functions in cell and tissue integrity certainly explain the increasing attention given to IF structural characteristics, dynamics and functions. IFs are progressively recognized as a key element of the cytoskeleton providing versatile, tunable, cell-type specific filamentous networks with unique cytoplasmic and nuclear functions.

\section{Main text}

\section{1-Working towards the elucidation of IF physiological functions.}

Intermediate filament are formed by a wide variety of proteins encoded by 73 genes in humans. With the exception of nuclear lamins found in all cells of multicellular organisms, the expression of IF proteins such as keratins, desmin or vimentin is cell-type specific and also varies during cell differentiation (Fig. 1). IF-related disease affect particular tissues or organs which mostly correspond to the sites of expression of the corresponding IF proteins. More than 90 pathologies, including the so-called laminopathies, keratinopathies and desminopathies, have been associated to IF protein mutations. When mutated, desmin, which is specifically expressed in muscle cells, impact muscle function causing skeletal myopathies or cardiomyopathies. In contrast, keratins which form a large family of IF proteins differentially expressed as sets of one, two, or three pairs in diverse epithelia give rise to several skin, hair lens or liver disorders.

In contrast, knock-out animal models have not always brought clear evidence of IF functions, possibly because of the complexity of the protein expression patterns and of functional redundancy. A major initial publication from Colucci-Guyon et al. (1994) claimed that loss of vimentin, a major IF protein expressed in all mesenchymal cells had no phenotypical impact in vivo. However, further studies have shown that vimentin actively participates in a wide range of cell, tissue and organ functions. Loss of vimentin induces a decrease of Bergmann glial cells and Purkinje cells in the cerebellum together with a deficit in motor coordination, a compromised endothelial integrity with increased arterial stiffness, a delayed lymphocyte and fibroblast migration during wound healing. Vimentin is also critical in pathological situations and, for instance, provides a protection against 
bacterial meningitis. Mice models with various IF deficiencies confirm the essential role of IFs in tissue physiology. Loss of keratins induces early embryonic lethality, requiring the use of conditional knock-out to gain further insight in their function in adults. GFAP knock-out mice are more sensitive to percussive head injuries and display altered astrogliosis and wound healing. Other examples include desmin whose deletion dramatically affects skeletal, smooth and cardiac muscle formation and synemin which plays a structural role in bone formation and mechanical resistance. These in vivo observations show that IF deletions cause alterations that are different from those observed in human diseases. In fact, IF-related diseases are caused by mutations which generally lead to the reorganization but not the loss of the IF network. For instance, the heterozygous mutations on the type III GFAP gene causing Alexander disease engender the accumulation of IF proteins in proteinaceous aggregates -called Rosenthale fibers- in astrocytes (Fig. 4). More specific mouse models expressing mutated IF proteins have confirmed that the altered organization of the IF network plays a key role in these pathologies. Finally, IF-related deseases and in vivo models point to IFs as essential in tissue mechanical resistance and tissue integrity.

Human genetic disorders demonstrate the importance of IF physiological functions. They speak for the need to understand IF role at the cellular and tissue scale but also underline the complexity of the task. Because of the high number of possibly redundant IF proteins, the wide variety of expression patterns, the intrinsic stability and the lack of specific inhibitors, the identification of IF functions have lagged behind that of the two other cytoskeletal networks, actin and microtubules. Our understanding of IF functions at the cellular and tissue scales has emerged from the careful and still on-going characterization of IF molecular structure. Building on the unique structural properties of IF proteins, the characterization of IF mechanical properties and the progressive elucidation of the regulatory mechanisms controlling IF's dynamics in cells, we now better grasp the extend of IF cell functions which eventually explain IF-related pathologies.

\section{2- Composition, assembly, structure and intrinsic properties of IFs}

\section{IF proteins}

IF proteins derive from a common ancestor of the more ubiquitously expressed nuclear lamins, traced back to species outside of the metazoan lineage like the slime mold Dictyostelium. IF proteins have a uniform global structure based on a common central domain. The first IF structure was discovered in 1932 by William Astbury using Xray diffraction and crystal structure theory and led, through Linus Pauling's alpha helix model, to the prediction of the coiled-coil structure of keratin by Francis Crick in 1952. This common $\alpha$-helical rod domain of approximately 310 amino acids is present in all IF proteins. It is flanked by head and tail domains of diverse size and structure which characterize each IF protein and allow them to interact with specific intracellular partners. Depending on their distinct rod domain sequence and different terminal domain functions, IF proteins are classified into six categories (Fig 1) which differ in the biochemical properties and their ability to associate with one another.

\section{IF assembly}

To form filaments, IF proteins first assemble into dimers through the rod domain of two monomers that are twisted together in a coiled-coil structure. For cytoplasmic IFs, dimers then associate in an antiparallel fashion to form apolar tetramers that constitute the soluble building blocks of the filaments. Eight tetramers join laterally to give rise to an Unit Length Filament (ULF) of approximately $50 \mathrm{~nm}$ length (Fig. 2). ULFs assemble end-to-end to constitute a filament with a diameter of $16 \mathrm{~nm}$ which is radially compacted to form the classical $10 \mathrm{~nm}$ diameter filament observed in cells. Notably, nuclear IFs composed of lamins form by concomitant lateral and longitudinal association of the dimers, giving rise to filaments with variable diameter and length. In 
all cases, IFs, contrasting with microfilaments and microtubules, polymerize independently of nucleotides, or protein nucleators. Instead, IF dynamics involves fusion and fission of ULFs or small pre-existing filaments (also called squiggles) and the exchange of subunits. However the extent of such events in cells is not clear and their regulation remains to be elucidated. The structural diversity of IFs is also not fully characterized. While keratins exclusively form type I/type II heterodimers, how type III IF proteins self-assemble to form homopolymers and also co-polymerize with other type III or type IV proteins? These co-assembly can involve 4 or 5 different IF proteins like observed in astrocytes where GFAP, vimentin, nestin and synemin are observed in the same filaments or in neurons where neurofilament proteins NF-L, NF-M, NF-H can interact with $\alpha$-internexin or peripherin. Do different IF proteins assemble in heteropolymers by forming common or distinct dimers, tetramers or ULFs which then assemble to form a single filament?

To the initial diversity in IF genes must also be added the large number of IF protein splice variants Keratin, vimentin, desmin, lamin, peripherin and GFAP are all alternatively spliced, and some of them have multiple splice variants, like GFAP which has more than 10 splice variants that can have different impact on the polymerization of the filaments. Finally, a variety of posttranslational modifications such as farnesylation, glycosylation, transglutamination, acetylation, ubiquitination, sumoylation and phosphorylation can occur on IF proteins (Fig. 3). The complex combination of post-translational modifications decorating IFs is likely associated with a wide range of dynamic and functional properties.

\section{IF mechanical properties}

Because of their core structure built on their rod domains and their auto-assembly, IFs are extremely flexible, stretchable and also resistant to cold, high ionic concentrations, and mechanical breakage. For instance, desmin IFs found in muscle cells can be stretched in vitro about $250 \%$ before breakage. When submitted to forces the coiled-coil $\alpha$-helical domain of each monomer can be individually stretched to eventually transition to a $\beta$-sheet conformation. The remaining $\alpha$-helical domains within a ULF allow it to return to its original length during relaxation. When submitted to large deformations in vitro IFs have an extraordinary ability to stiffen and can therefore withstand large strains without breaking. Strain-stiffening is also observed with lamin filaments which deform reversibly at a few hundred picoNewtons and stiffen at nanoNewton forces. The stiffening of the filament is attributed to an increased pull on beta sheets. The softness of IFs observed under compression also leads to a progressive stiffening of the filaments which, however, cannot buckle unlike actin fibers and microtubules.

IF structure appears at the core of their unique mechanical properties in vitro. As we are still missing some essential information about the structure of complex IF polymers in cells, we can only guess that IF composition, alternative splicing and post-translational modification influence the mechanics of IFs. However, analyses of IF's functions in cells progressively reveal how IF networks participate to cell and tissue integrity.

\section{3- IFs as integrators of intracellular space.}

\section{Resilient intracellular scaffolds}

An important characteristic of most IF-related disease is that the mutations do not prevent the protein expression but instead induce the aggregation or the collapse of the network in a perinuclear area. This points to an essential role of IF network organization within cells. In mammalian cells, IFs form at least two distinct intracellular networks; the cytosolic and the nuclear networks (Fig. 2). Because IF proteins of different types cannot always co-assemble, cells can sometimes display two distinct cytoplasmic IF networks. For instance, distinct type III IFs like vimentin IFs and keratin IFs 
can be found in epithelial cells (Fig. 2). Cytoplasmic and nuclear IFs form scaffolding structures which resist and also adapt to the physical challenges of their environment. Cytoplasmic IFs extend between the periphery of the cell and the nucleus, which they often surround forming a so-called perinuclear cage. Nuclear lamin polymers form the nuclear lamina, a protein meshwork that supports the nuclear envelope structure and tethers heterochromatin to the nuclear periphery.

Because of their high robustness and elastic properties IFs are excellent stress absorbers and key for cell mechanical resilience. This is reflected by their essential contribution to the regulation of nuclear and cell stiffness under deformations. Under small deformation, IFs make cells compliant, while under large stress and deformation, IFs provide cells with mechanical strength and stiffness. Overall, the IF networks are essential actors of the cell intrinsic mechanical resilience. The expression pattern of IF proteins in a given cell type may tune the cell mechanical properties to the specific tissue environment. Differences in amino acid sequence, as observed between different IF proteins or mutant variants, or post-translational modifications can change the charge and hydrophobicity of the IF proteins. In turn, this affects the stretching and sliding of monomers within the filaments and may account for the variation in flexibility observed between IF types. As an example, neurofilaments tend to be much softer than other IFs, which may be related to their expression in brain tissues that are particularly soft. The role of IFs in cell mechanics is particularly important in tissues submitted to frequent mechanical stresses such as striated muscles, heart, skin or blood vessels. Mutations in keratin 5 and keratin 14, causing Epidermolysis Bullosa Simplex, disrupt the mechanical integrity of the epidermis and causes blistering, and desmin mutations affect the integrity of skeletal and cardiac muscle tissues.

\section{Mechanical and functional support for intracellular organelles}

The intracellular scaffolds formed IFs globaly influence organelle position and dynamics by participating to the viscosity of the cytoplasm. However, IFs have a more direct role in the organization of the cellular space as they directly interact with intracellular organelles. As a consequence, IFs are responsible for the positioning and the number of autophagosomes and lysosomes during autophagy and play a role in the distribution of melanosomes. They also influence both anterograde and retrograde vesicular transports by controlling the position of intracellular organelles. Alteration of the IF network or IF mutations not only affect the distribution of organelles but also their associated signaling pathways, eventually leading to a loss of specific function. The role of IFs in the localization, structure and function of intracellular organelles is best illustrated by IF interaction with mitochondria. IFs surround mitochondria, either by confinement or by binding the mitochondrial membrane via the protein plectin. Mitochondria fulfill essential roles in ATP production, metabolic regulation, calcium signaling, generation of re active oxygen species (ROS), products of oxidative stress, and in cell apoptosis. Defects in mitochondrial distribution, morphology and functions are observed in several IF-related diseases such as Charcot-Tooth neuropathy or epidermolysis bullosa simplex. Cytoplasmic IFs protect mitochondria against ROS shedding light on the causes of oxidative stress observed in these disorders.

Both nuclear and cytoplasmic IFs interact with the nuclear enveloppe through the LINC complex, an assembly of proteins anchoring the cytosolic cytoskeleton to the inner meshwork of lamins. The cage-like structure of IFs around the nucleus actively participates in the control of nuclear shape and positioning. The dense and elastic cytoplasmic IF network contributes to the transmission of external forces applied on the cell cortex to the nucleus. In skeletal muscles, nuclei increase their length and aspect ratio upon cell stretching. The stretching capability of the nucleus relies on the desmin network which transmits force towards the myofibrillar nuclei. The IF perinuclear cage also protects the nucleus from excessive forces. When cells migrate through tight constrictions that squeeze the nucleus, vimentin IFs prevent nuclear rupture and DNA damage. 
Vimentin levels also control chromatin condensation, suggesting a direct link between nuclear deformations and the control of gene expression. The possibility that cytoplasmic IFs enter the nucleus is a matter of debate. Keratins such as $\mathrm{K} 17$, have been shown to interact with nuclear proteins and to impact nuclear morphology, histone modification, chromatin organization, gene expression, and proliferation in epithelial cells.

The intranuclear meshwork of lamins play a fundamental role in nuclear shape and mechanics and lamins elastic capabilities are crucial to uphold nucleus integrity during cell compression. Lamins also impact the dynamic of chromatin in the interphase nucleus and may thereby influence nuclear functions. Because of their tight interaction with the nuclear envelop, and their unique mechanical properties, IFs serve as protectors of nuclear integrity and probably also influence the mechanosensitive organization and functions of the nucleus. In skeletal muscle cells lamin A mutations related to muscular dystrophy cause transient rupture of the nuclear enveloppe, resulting in DNA damage, DNA damage response activation and reduced cell viability. Moreover, like cytoplasmic IFs, lamins are also important in the protection of the cell and the nuclei against oxidative stress. Upon induction of oxidative stress, a higher percentage of ROS is found in patient fibroblasts expressing mutated lamin A.

\section{4- IFs as integrators of cell mechanical properties}

\section{Cytoskeletal connections}

The structural and mechanical functions of IFs are reinforced by their tight connection and crosstalk with the actin and microtubule networks. This is perfectly illustrated in muscle fibers, in which desmin filaments connect the Z-discs to the plasma membrane, the mitochondria and the nuclei to increase the mechanical resilience of muscle cells. In addition to a possible direct interaction with actin microfilaments, a wide variety of cytoskeletal crosslinkers connect IFs to actin and microtubules. These include plectins, beta-crystallin, fimbrin, filaminA, APC (Adenomatous Polyposis Coli), as well as a number of cytoskeletal motors.

The impact of IFs is not only the result of their physical interactions with actin and microtubules but also involves IF function in key regulatory signaling pathways including RhoGTPase signaling. IFs influence the dynamics and organization of the microtubule and actin networks. Accumulating evidence point to IFs are major regulators of actin stress fibers and acto-myosin mediated forces, although the effects vary with cell types, possibly as a consequence of differences in IF composition. Vimentin IFs also interact with microtubules. Although no major change in the global organization of the microtubule network has been observed in IF depleted cells, IFs can modulate the dynamics of microtubules. Vimentin IFs serve as a template and subtly direct microtubule growth. Microtubules that are directly associated with vimentin are also more resistant to microtubule depolymerizing drugs like nocodazole. Whether all types of IFs have the same effect on the other cytoskeletal networks needs to be further investigated. However, it is already clear that keratin and vimentin networks behave very differently in relation with microtubules, as microtubule depolymerization induces the perinuclear collapse of the vimentin IF network without affecting keratin IF organization. Overall, it is likeky that IF composition or post-translational modifications influence cell contractility and motile behavior and may be key to the adaptation of various cell types to the specific and possibly evolving mechanical properties of their environment.

\section{Interactions with adhesive structures}

The scaffolding structure formed by cytoplasmic IFs, together with the other cytoskeletal networks, is connected to the extracellular microenvironment. IFs interact with, are regulated by and also influence cell-cell and cell-extracellular matrix adhesive structures. This has been initially 
illustrated by the association of keratin filaments with desmosomes via desmoplakin and with hemidesmosomes via BPAG1/2 (Bullous pemphigoid antigens). Keratin IFs stabilize hemi-desmosomes and desmosomes, which are essential for the cohesion of epithelial tissues. The stabilizing role of IFs on cell adhesions is not limited to epithelial cells. IFs are involved in the stability of gap junctions in cardiomyocytes and of adherens junctions in endothelial cells. IFs also associate with focal adhesions in many cell types, including fibroblasts, endothelial cells and astrocytes. The organization of IFs at focal adhesions and the exact molecular link that connect IFs to integrins are not entirely clear and likely vary depending on the IF composition and on the integrin involved. One specific IF protein, synemin directly interacts with several focal adhesion proteins such as talin, vinculin, zyxin. In absence of synemin, plectin, and more specifically plectin 1f, has been involved in the association of vimentin and desmin with integrins. IFs tend to stabilize or reinforce focal adhesions in immobile cells. However, the molecular mechanisms physically and functionally bridging IFs to focal adhesions needs to be further investigated, focusing on the specific relationship of each IF proteins with various integrins and focal adhesion proteins.

\section{5- IFs as integrators of intracellular signaling}

IFs not only serve as a structural scaffold but also form a molecular scaffold which connects with signaling pathways that influence cell behavior in physiological and pathological situations. The connection of IFs with intracellular structures parallels IF's ability to influence cell functions. While focal adhesion-mediated signaling influences the organization of the IF network, IFs can in turn influence the dynamics of focal adhesions. They participate in focal adhesion-associated signaling, as shown for vimentin which regulates the expression level and the localization of the focal adhesion kinase (FAK) and of the Rac1-guanosine exchange factor Vav2. In addition to their role in controlling acto-myosin contractility, vimentin and more generally type III IFs facilitate cell migration and invasion of mesenchymal cells by controlling the dynamics, the distribution of focal adhesions. Depending on the cell types, IFs have different effect on cell migration which may be explained by the difference in IF proteins or integrin expression patterns.

Mirroring their protective role against mechanical stresses, the signaling functions of IFs are also involved in cellular survival by promoting cell cycle progression, maintaining organelle homeostasis and protecting from apoptosis. Most IF proteins including keratins, GFAP, vimentin and neurofilaments interact with 14-3-3 proteins. The 14-3-3 family of proteins forms a major class of molecular chaperones, binding several kinases including Raf, PKC, c-Bcr and BcrAbl, and PI3kinase and thereby regulating their activity, their localization or their stability. By controlling 14-33 localization, IFs are well positioned to influence a wide range of vital regulatory processes, such as mitogenic signal transduction, apoptotic cell death, and cell cycle control. 14-3-3 interactions with target proteins may participate in the formation of protein aggregates, such as those observed during neurodegenerative diseases, and may regulate pathogenic processes. Other signaling functions have been described for keratin IFs. Keratin K8/K18 IFs interact with the cytoplasmic tail of TNF receptors to influence their signaling and protect cells from apoptosis. The same K8/K18 have also been shown to interact with and to activate Notch1 signaling and promote epithelial differentiation in the large intestine. However, in the case of Notch regulation, vimentin can also play a crucial regulatory role. In endothelial cells vimentin regulates Notch signaling strength and arterial remodeling in response to hemodynamic forces, suggesting that several IF proteins may contribute to the regulation of the Notch pathway. In the nucleus, lamins control nucleocytoplasmic transport and gene expression. The alteration of lamin in hungtintin-mediated neurodegeneration affects the associated chromatin domain, chromatin accessibility and transcriptional regulation. 
We are probably only scratching the surface when considering IF's involvement in biochemical signals. How the composition of IFs can modulate intracellular signaling is also a vast question, to which systematic proteomic analysis of IF molecular partners may help answer. In addition, recent evidence suggests the role of soluble IF proteins in IF signaling functions. Phosphorylated soluble lamins interact with gene enhancer regions to control gene expression, suggesting that local or partial depolymerisation of IFs may provide soluble proteins serving as signaling intermediate.

\section{6- Dynamic control of IF functions}

\section{Control of IF protein expression}

Changes in IF protein expression can modulate the mechanical properties of the network and consequently of the cells and tissues. Hundred to thousand times accumulation of keratin filaments in the cornified layer of the skin generates a material with an elastic modulus in the megaPascal range. Within the blood vessel network, vimentin levels are present at proportional levels with the size of the vessel. Indeed, with increasing shear stress due to heavier blood flow in large arteries, the endothelial cells and vascular smooth muscle cells need more resistance to the pressure. Changes in the composition of the network generally parallel cell differentiation and allows for important but relatively slow modifications of the cell and tissue mechanics. During epithelial to mesenchymal transition, vimentin expression increases and facilitates cell shape changes and cell invasion (Fig. 5A). IF protein expression is controled at the transcriptional level but also involve the regulation of IF protein stability. Ubiquitination targets IF proteins for degradation, a process that is crucial for the correct turnover of the IF network as illustrated by giant axonal neuropathies in which mutations of the E3 ligase targeting factor gigaxonin leads to an accumulation of IFs in the central nervous system. Until now, there is no clear evidence that the network composition can be tuned at a subcellular level, and therefore such changes in protein expression may only allow for global longterm changes in the properties of the network.

\section{Control in time}

Active rearrangements of the IFs including polymerization, depolymerisation, aggregation, transport or bundling of filaments modify the meshsize and the mechanical properties of the network. In hair, nail, hoof and quill, keratin IFs are crosslinked by disulfide bonds giving rise to materials with an elastic modulus in the GPa range. In epithelial cells, the meshsize of the keratin network tend to increase towards the cell periphery, providing a less elastic network which might facilitate cell protrusion and motility. Upon shear stress, the meshsize becomes more homogeneous throughout the cell, and generate a more elastic and protective intracellular structure. The control of the meshsize indicates that intracellular signals can modify IFs as cells changes behavior or adapt to different microenvironments. Dramatic rearrangements of the IF network are indeed observed as cells respond to external stresses, divide, migrate or die (Fig. 5). IF organization dramatically changes when cell morphology and functions require an adaptation of cell mechanical properties and the reorganization of the cytoplasm. During cell division (Fig. 5B) the redistribution of the IF network affects the organization of the cortical actin network and contribute to mitotic progression. Remodeling of the IF network during cell cycle progression and mitosis is mostly triggered by phosphorylation of IF proteins, in particular by Cyclin Dependent Kinases (Cdks). Such kinases often phosphorylate IF proteins and alter their filamentous organization, rendering them more (or less) soluble. Phosphorylation can similarly promote the disassembly of the nuclear lamina and thus promote nuclear envelope breakdown. External stress including heat stress, osmotic stress, mechanical stress generally induce the hyperphosphorylation of IF proteins and the reorganization of the network (Fig. 5C). This is frequently associated with the binding of IFs to Heat Shock Proteins although different IFs have overlapping or selective HSP binding partners. These observations led 
to the hypothesis that IFs may protect the cell from stress-induced death by passively buffering the activity of stress-activated kinases. However our more recent understanding of the functions of the IF network and their regulation by intracellular signaling suggest that stress-induced changes in IF organization may play an active role in adapting cell mechanics, intracellular organization and functions of key organelles to maintain cell homeostasis. During apoptosis, the cytoplasmic and nuclear IF networks are totally disrupted and collapse in large aggregates as proteins are irreversibly cleaved by caspases, which allows the cell to shrink and die. Kinases. Together with changes in IF composition, reversible or irreversible post-translational modifications and cytoskeletal coupling, the IF network adapts to major changes in the cell environment or cell behavior. More subtle rearrangements of the IF network can occur at a subcellular level and contribute to local adjustment of the scaffolding properties of the IF network.

\section{Control in space}

Signaling cascades downstream of membrane receptors can locally influence the dynamics and the properties of IFs. The role of RhoGTPases and their effectors PAK and ROCK in IF protein phosphorylation hints at how intracellular signaling induced may locally influence IF dynamics, organization and mechanical properties in cells. Modulation of RhoGTPase signaling downstream of integrins for instance, can alter the organization of the IF network. In migrating epithelial cells, the global redistribution of polymerization sites towards the leading edge facilitates the generation of new filaments and organizes the turnover of the network. In contrast, in migrating astrocytes the polarization of the IF network relies on an increased kinesin-mediated transport of vimentin and GFAP-containing IFs towards the front edge coupled to a local integrin-dependent Cdc42-driven inhibition of their dynein-mediated retrograde transport. Focal adhesion-mediated signaling also regulate vimentin phosphorylation. Fibroblasts plated on soft substrates show an increase in vimentin phosphorylation associated with an increase in the soluble pool of proteins suggesting an adaptation of the properties of the network to the mechanical characteristics of the cell microenvironment. Cell stretching has been recently shown increase the tension of keratin fibers thereby controlling their interaction with cten/tensin 4, to influence focal adhesion signaling.

In parallel to their effects on IF solubility and mechanical properties, post-translational modifications can also modify the molecular interactions of IF proteins and thereby influence intracellular signaling. This has been nicely illustrated in the case of 14-3-3 proteins which specifically bind to phosphorylated keratins K8/K18 IFs. The control of 14-3-3 localisation by phosphorylation of keratins or vimentin strongly influences cell signaling. During S and G2 phase, keratins become hyperphosphorylated which results in an increased soluble pool of IFs that sequesters 14-3-3 proteins away from the Cdc25. Dephosphorylation of keratins releases 14-3-3 as IFs return to their G1 filamentous states. Interestingly, recent observations suggest that the stable lamin network can dissociate from the lamina and become diffuse in the nucleoplasm depending on substrate rigidity. This observation opens exciting avenues for exploration. For example, the identification of regulatory mechanism controling lamin organization may help us understand the consequences of such rearrangements in nuclear organization and possibly unveil new pathways controlling gene expression.

Combined with their intrinsic mechanical properties, the connection of the IF network with most cellular structures place makes them excellent candidates to mediate mechanical cell responses, possibly tuning cellular functions including gene regulation by transmitting or protecting from both external or internal forces/mechanical strains. 


\section{Conclusions}

The unique structural properties of IFs are at the core of their mechanical and signaling functions. As intracellular scaffolds, the cytoplasmic and nuclear IF networks integrate intracellular space, cell mechanics, intracellular signaling to protect cells from physical, chemical or biological stresses. Cytoplasmic and nuclear IFs not only serve as guardians of intracellular organization, cell integrity but also actively participate in signaling cascades to promote cell cycle progression, cell survival or to inhibit apoptosis. Overall, IFs play an essential role in cell and tissue resilience, maintaining their integrity during development and throughout the multiple physical, chemical or biological challenges encountered by living organisms. Most importantly, the dynamic control of IF protein expression and post-translational modifications modifies the mechanical and signaling properties of the IF network. This trigger cellular responses to environmental challenges allowing cells and tissues to continuously adapt to their environment.

\section{Acknowledgements}

This work was supported by the La Ligue contre le cancer (S-CR17017), Centre National de la Recherche Scientifique and Institut Pasteur. GDP is funded by the Pasteur International PhD Program and is enrolled at Sorbonne University ED515. We would like to thank Jean-Baptiste Manneville for critical reading of the manuscript.

\section{Declaration of interests}

The authors declare no competing interests.

\section{Further readings}

Alcala-Vida, R., M. Garcia-Forn, C. Castany-Pladevall, J. Creus-Muncunill, Y. Ito, E. Blanco, A. Golbano, K. Crespi-Vazquez, A. Parry, G. Slater, S. Samarajiwa, S. Peiro, L. Di Croce, M. Narita, and E. Perez-Navarro. 2021. Neuron type-specific increase in lamin B1 contributes to nuclear dysfunction in Huntington's disease. EMBO Mol Med. 13:e12105.

Charrier, E.E., A. Asnacios, R. Milloud, R. De Mets, M. Balland, F. Delort, O. Cardoso, P. Vicart, S. Batonnet-Pichon, and S. Henon. 2016. Desmin Mutation in the C-Terminal Domain Impairs Traction Force Generation in Myoblasts. Biophys J. 110:470-480.

De Pascalis, C., C. Pérez-González, S. Seetharaman, B. Boëda, B. Vianay, M. Burute, C. Leduc, N. Borghi, X. Trepat, and S. Etienne-Manneville. 2018. Intermediate filaments control collective migration by restricting traction forces and sustaining cell-cell contacts. The Journal of Cell Biology. 217:3031-3044.

Duarte, S., A. Viedma-Poyatos, E. Navarro-Carrasco, A.E. Martinez, M.A. Pajares, and D. Perez-Sala. 2019. Vimentin filaments interact with the actin cortex in mitosis allowing normal cell division. Nat Commun. 10:4200.

Earle, A.J., T.J. Kirby, G.R. Fedorchak, P. Isermann, J. Patel, S. Iruvanti, S.A. Moore, G. Bonne, L.L. Wallrath, and J. Lammerding. 2020. Mutant lamins cause nuclear envelope rupture and DNA damage in skeletal muscle cells. Nature materials. 19:464-473.

Forsting, J., J. Kraxner, H. Witt, A. Janshoff, and S. Koster. 2019. Vimentin Intermediate Filaments Undergo Irreversible Conformational Changes during Cyclic Loading. Nano letters. 19:7349-7356.

Ikegami, K., S. Secchia, O. Almakki, J.D. Lieb, and I.P. Moskowitz. 2020. Phosphorylated Lamin A/C in the Nuclear Interior Binds Active Enhancers Associated with Abnormal Transcription in Progeria. Dev Cell. 52:699-713 e611.

Jacob, J.T., R.R. Nair, B.G. Poll, C.M. Pineda, R.P. Hobbs, M.J. Matunis, and P.A. Coulombe. 2020. Keratin 17 regulates nuclear morphology and chromatin organization. J Cell Sci. 133. 
Jiu, Y., J. Peranen, N. Schaible, F. Cheng, J.E. Eriksson, R. Krishnan, and P. Lappalainen. 2017. Vimentin intermediate filaments control actin stress fiber assembly through GEF-H1 and RhoA. $J$ Cell Sci. 130:892-902.

Lahdeniemi, I.A.K., J.O. Misiorek, C.J.M. Antila, S.K. Landor, C.A. Stenvall, L.E. Fortelius, L.K. Bergstrom, C. Sahlgren, and D.M. Toivola. 2017. Keratins regulate colonic epithelial cell differentiation through the Notch1 signalling pathway. Cell Death Differ. 24:984-996.

Leduc, C., and S. Etienne-Manneville. 2017. Regulation of microtubule-associated motors drives intermediate filament network polarization. J Cell Biol. 216:1689-1703.

Mucke, N., L. Kammerer, S. Winheim, R. Kirmse, J. Krieger, M. Mildenberger, J. Bassler, E. Hurt, W.H. Goldmann, U. Aebi, K. Toth, J. Langowski, and H. Herrmann. 2018. Assembly Kinetics of Vimentin Tetramers to Unit-Length Filaments: A Stopped-Flow Study. Biophys J. 114:2408-2418.

Patteson, A.E., A. Vahabikashi, K. Pogoda, S.A. Adam, K. Mandal, M. Kittisopikul, S. Sivagurunathan, A. Goldman, R.D. Goldman, and P.A. Janmey. 2019. Vimentin protects cells against nuclear rupture and DNA damage during migration. J Cell Biol. 218:4079-4092.

Robert, A., M.J. Rossow, C. Hookway, S.A. Adam, and V.I. Gelfand. 2015. Vimentin filament precursors exchange subunits in an ATP-dependent manner. Proc Natl Acad Sci U S A. 112:E35053514.

Sapra, K.T., Z. Qin, A. Dubrovsky-Gaupp, U. Aebi, D.J. Muller, M.J. Buehler, and O. Medalia. 2020. Nonlinear mechanics of lamin filaments and the meshwork topology build an emergent nuclear lamina. Nat Commun. 11:6205.

Steen, K., D. Chen, F. Wang, R. Majumdar, S. Chen, S. Kumar, D.B. Lombard, R. Weigert, A.G. Zieman, C.A. Parent, and P.A. Coulombe. 2020. A role for keratins in supporting mitochondrial organization and function in skin keratinocytes. Mol Biol Cell. 31:1103-1111.

Szeverenyi, I., A.J. Cassidy, C.W. Chung, B.T. Lee, J.E. Common, S.C. Ogg, H. Chen, S.Y. Sim, W.L. Goh, K.W. Ng, J.A. Simpson, L.L. Chee, G.H. Eng, B. Li, D.P. Lunny, D. Chuon, A. Venkatesh, K.H. Khoo, W.H. McLean, Y.P. Lim, and E.B. Lane. 2008. The Human Intermediate Filament Database: comprehensive information on a gene family involved in many human diseases. Human mutation. 29:351-360.

van Engeland, N.C.A., F. Suarez Rodriguez, A. Rivero-Muller, T. Ristori, C.L. Duran, O. Stassen, D. Antfolk, R.C.H. Driessen, S. Ruohonen, S.T. Ruohonen, S. Nuutinen, E. Savontaus, S. Loerakker, K.J. Bayless, M. Sjoqvist, C.V.C. Bouten, J.E. Eriksson, and C.M. Sahlgren. 2019. Vimentin regulates Notch signaling strength and arterial remodeling in response to hemodynamic stress. Scientific reports. 9:12415. 


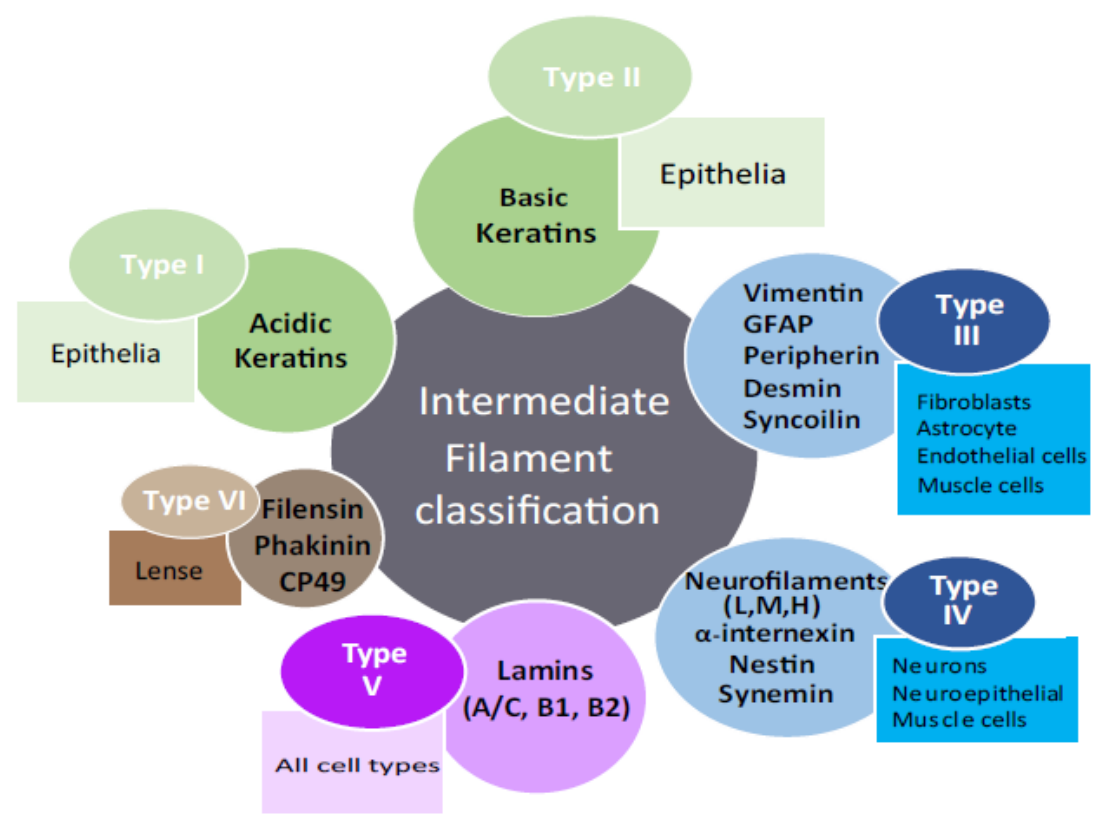

Intermediate filament classification: The type I and II correspond to acidic and basic keratins the most abundant form of IF proteins, which associate in obligate heteropolymers that are highly expressed in epithelial cells. The Type III proteins are expressed in a variety of cell types, including fibroblasts, endothelial, glial and muscle cells and comprise vimentin, desmin, GFAP, as well as peripherin. Type IV proteins including nestin, synemin, $\alpha$-internexin (a-Inx), syncoilin and neurofilaments are majorly expressed in progenitor or differentiating cells and also in neuron and glial cells. Type $\mathrm{V}$ proteins correspond to the ubiquitously expressed lamins which contain an NLS and a CAAX box motif that targets the protein to the inner nuclear membrane. Finally, type VI is a group of very divergent proteins which includes the lensspecific IF proteins (Bfsp1 also known as filensin, and Bfsp2, also known as phakinin and CP49). 


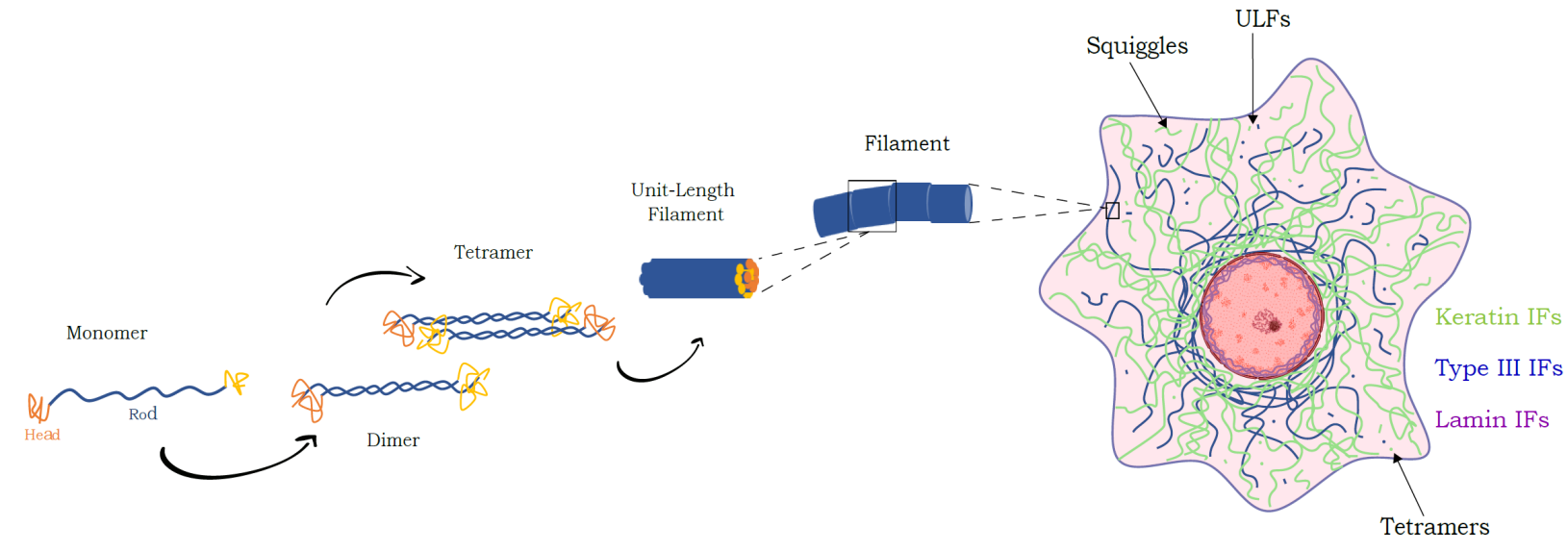

IFs assembly: Monomers of IFs assemble into dimer by interaction of their rod domains. Dimers assemble in an antiparallel manner to form soluble tetramers. Tetramers then assemble into Unit Length Filaments which via end-to-end binding elongate into filaments. Soluble tetramers, ULFs, squiggles which correspond to short IFs and long IFs can all be found in cells.

Figure 3

Vimentin post-translational modifications

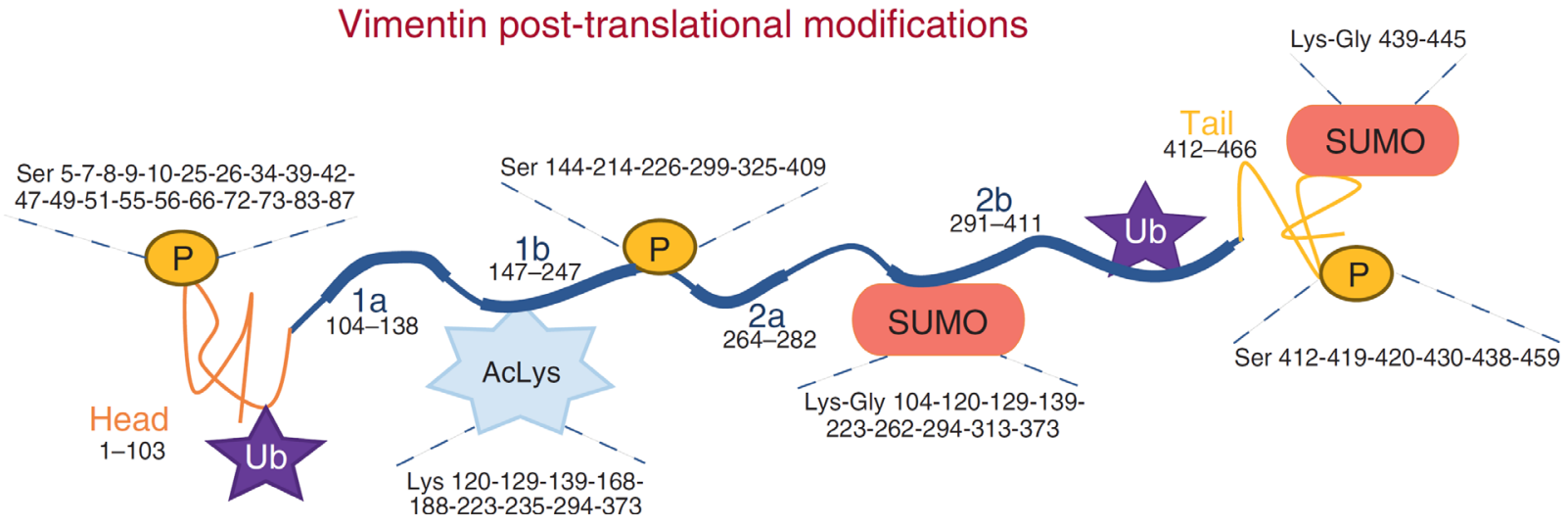

IF post-translational modifications (PTM): The schematics depicts the major posttranslational modifications, including for phosphorylation, sumoylation, acetylation and ubiquitination, known to affect vimentin. As for all IF proteins, PTM occurs throughout the proteins and affects the dynamics, the mechanics and the biochemical properties of IFs. 


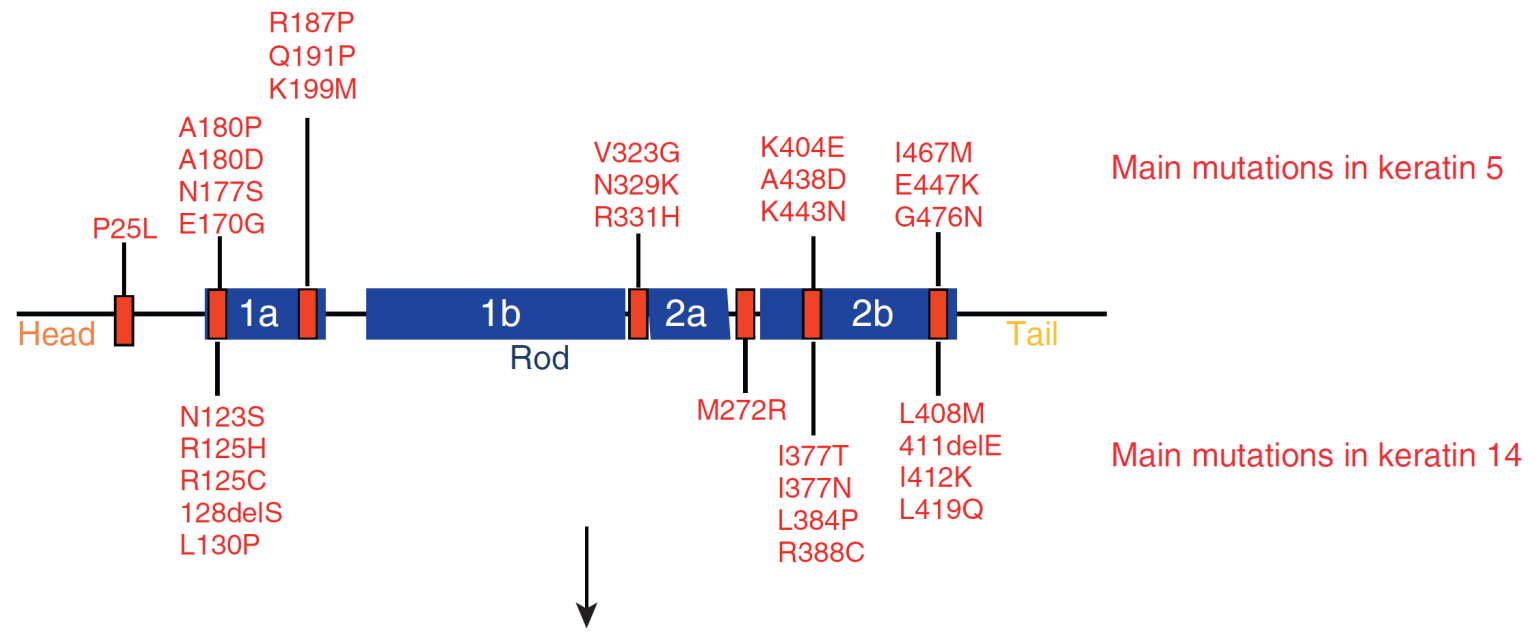

Aggregation of keratin epidermolysis bullosa simplex

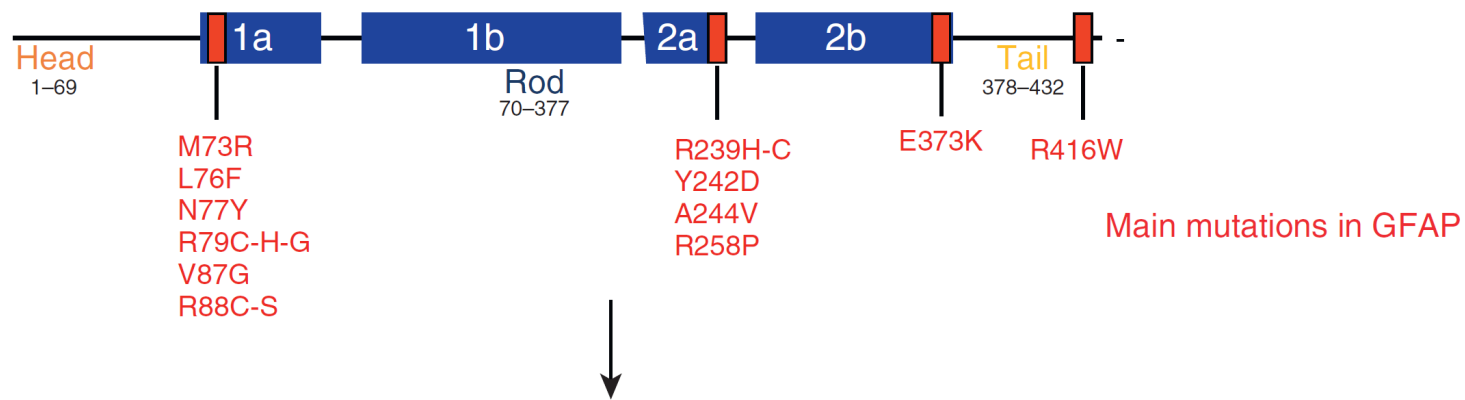

Aggregation in Rosenthale fibers (Alexander disease)

IF mutations are responsible for human diseases: The schematics shows the main mutations (in red) observed in Keratin 5/14 and GFAP leading to disease. Mutations of K14 and K5 lead to EBS disorder and GFAP mutation to Alexander disease. 


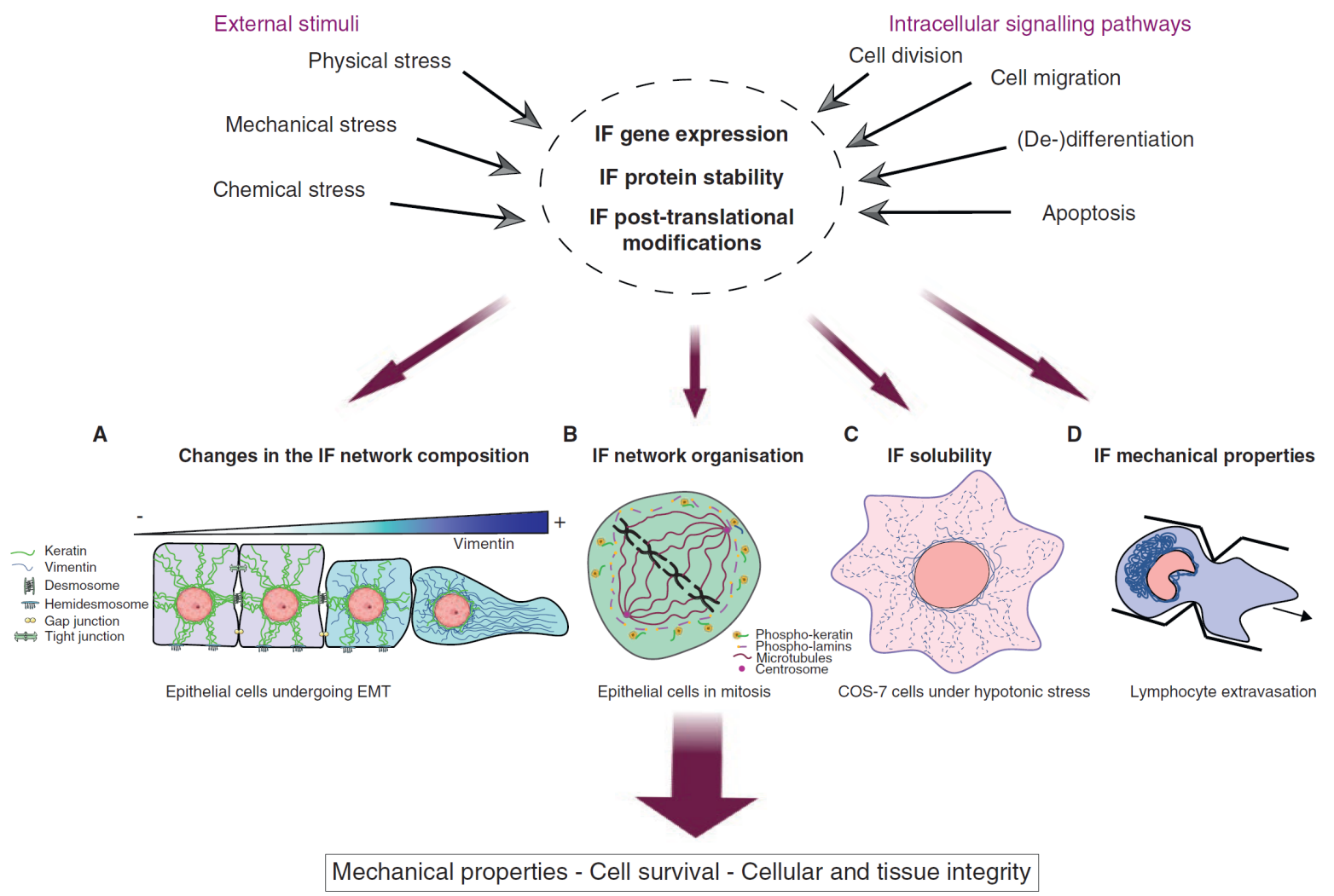

External and intracellular signals lead to changes in IFs to ensuring cellular mechanical stability and tissue integrity: Cells are subjected to external stimuli and intrinsic changes that modify the expression, the stability and the post-translational modification of IFs. These changes affect the composition and the organization, the mechanical and biochemical properties of the IF network. A Epithelial-Mesenchymal Transition (EMT) where keratin in epithelial cells is progressively replaced by a vimentin network as the cell transition to a migrating mesenchymal type facilitating tissue invasion. B. Modification of the cytoplasmic and nuclear network during cell division with the phosphorylation of cytoplasmic IFs (here keratins) and lamins. C.The phosphorylation of IFs leads to the solubilisation of the network in response to osmotic shock. D. The organisation of vimentin IF network is dramatically altered as lymphocytes migrate between endothelial cells during extravasation. 